\title{
Performance analysis and optimization of a box-hull wave energy converter concept
}

\section{Article}

\section{Accepted Version}

Creative Commons: Attribution-Noncommercial-No Derivative Works 4.0

Bódai, T. and Srinil, N. (2015) Performance analysis and optimization of a box-hull wave energy converter concept. Renewable Energy, 81. pp. 551-565. ISSN 0960-1481 doi: https://doi.org/10.1016/j.renene.2015.03.040 Available at https://centaur.reading.ac.uk/73381/

It is advisable to refer to the publisher's version if you intend to cite from the work. See Guidance on citing.

Published version at: http://dx.doi.org/10.1016/j.renene.2015.03.040

To link to this article DOI: http://dx.doi.org/10.1016/j.renene.2015.03.040

Publisher: Elsevier

All outputs in CentAUR are protected by Intellectual Property Rights law, including copyright law. Copyright and IPR is retained by the creators or other copyright holders. Terms and conditions for use of this material are defined in the End User Agreement.

\section{www.reading.ac.uk/centaur}

\section{CentAUR}

Central Archive at the University of Reading

Reading's research outputs online 


\title{
Performance analysis and optimization of a box-hull wave energy converter concept
}

Tamás Bódai ${ }^{\mathrm{a}, *}$, Narakorn Srinil ${ }^{\mathrm{b}}$

3

${ }^{a}$ Meteorological Institute, University of Hamburg, Hamburg, Germany

${ }^{b}$ Department of Naval Architecture, Ocean and Marine Engineering, University of Strathclyde, Glasgow, $U K$

\begin{abstract}
In this paper we consider a wave energy converter concept which is created by linking a box barge to the mechanical reference by linear dampers. The response to incident wave action in terms of power take-off is expressed explicitly as the solution of a linear frequencydomain model. The simplicity of the model combined with the possibility of the application of theory allows for a nested, and so manageable, procedure of optimization. We find that for any geometry, i.e., a combination of e.g. the breadth-to-length and breadth-to-draught aspect ratios of the box, the optimum is characterized by resonance at least in one of the two degrees of freedom, heave or pitch. Furthermore, optimal geometries turn out to be extremal: either long attenuator-type or wide terminator-type devices perform the best. We find also that optimal wavelengths, which are comparable to the device length in case of attenuators, emerge either due to the progressively increasing buoyancy restoring force characteristic, or due to the finite bandwidth of irregular waves. In particular, diffraction forces are more significant under optimal conditions for performance in irregular seas in comparison with conditions necessary for the most intensive displacement response of the free-floating box barge exposed to regular waves.
\end{abstract}

Keywords: wave energy converter, optimization, box barge, radiation-diffraction analysis, dimensional analysis

\section{Introduction}

For economic viability the optimization of wave energy converters (WEC) has to satisfy extreme requirements $[1,2]$. It has been recognized to be a difficult task in comparison with other types of renewable energy. For the survival and ultimate success of the industry it needs a higher degree of knowledge sharing at this early stage. Furthermore, in creating a knowledge base for the industry, beyond the examples of individual devices with their

${ }^{*}$ Corresponding author

Email addresses: tamas.bodai@uni-hamburg.de (Tamás Bódai), narakorn.srinil@strath.ac.uk (Narakorn Srinil) 
'development narratives', aspects of general interest should be considered too. Such fundamental aspects and the related theoretical analysis in most cases require abstraction and simplification.

This paper presents a procedure for the optimization of an archetypal concept. A sketch of the concept can be seen in Fig. 1, which also depicts the level of realism at which we intend to address the problem of optimization. The floating part of the device concept is a large box barge, whose free-floating motion was previously studied by Kraemer [3] with a focus on the combined effects of wave frequency and wavelength. He made an attempt to find optimal values of these, corresponding to the most intensive response, relative to the natural frequency and device length, respectively. Outstanding questions remain, however, whether the maximal amplitude of oscillation in pitch entails maximal power take-off (PTO) when the PTO mechanism is represented very simply by a linear damper, and if the conditions of optimum remain the same when varying other geometrical parameters that determine performance.

In our study we are concerned with what can be viewed as the preliminary steps of the development process of a WEC. We confine our analysis to the simple settings of small amplitude 2-dimensional long-crested waves of an idealized frequency spectrum. We take the most idealized picture of a PTO mechanism, a linear damper of constant characteristics, i.e., no PTO control is considered. However, studies considering PTO control $[4,5,6,7,8,9,10]$ show a promising potential for enhancing performance. Furthermore, the very simple box hull-shape has been chosen in favor of the possibility of having a theoretical understanding. A mooring system would also be inevitable for a working engineering construction in many cases $[11,12]$; and the list of further engineering compromises, which could impact strongly on performance, could be continued. Furthermore we note that this study takes a point of view that optimal design and environmental parameters together are searched for, with a view of subsequently finding suitable sea sites for a device. The alternative would be to first make a choice of a certain sea site, and then optimize the design parameters only, so as they best suit that environment [13]. That is, we extend the settings defined by Kraemer mainly by two components: (i) adding a PTO mechanism, and (ii) considering a wave spectrum.

A link of the float with the mechanical reference (i.e., Earth's inertial frame of reference) is an ideal choice in that - if given the same hull geometry - no other PTO mechanism is expected to outperform this one. In a 2-dimensional (2D) setting, when the float is confined to moving in a vertical plane only, the regular wave frequency and the damping coefficients of a 2 degrees-of-freedom (2-DOF) device can be set so that the incident waves are completely destructed, i.e., a $100 \%$ efficiency is theoretically possible $[14,15]$. This possibility has been recognized and experimentally demonstrated in case of other devices, such as: Salter's duck [16] and the Bristol cylinder [17]. If a link with the mechanical reference is not possible and power take-off has to rely on inertial forces, a modified PTO mechanism is expected to perform less effectively. In this case, however, one may adopt the following strategy to find an optimum. One can retain the geometry that was found optimal having a link with the mechanical reference, and then the objective is to tune the new PTO mechanism possibly involving inertial parts - in a way that it best mimics the effect of the ideal PTO mechanism [18]. These ideas might apply in case of the free-floating SEAREV device [8]. 
Optimization, that is, finding the maximum value of an objective function, can be difficult in practice for various reasons. One reason may be that multiple local optima exist, in which case with different initial guess values for the parameters to be tuned, different and possibly misleading results can be obtained. Another reason may be that the objective function cannot be resolved smoothly numerically, which could challenge many optimization algorithms. Any such difficulties are enhanced by larger number of variables to be optimized. In this case, if various types of algorithmic or automatic optimum searches fail or provide inconsistent results, the manual treatment (as well as the troubleshooting) might be beyond the possibilities. Even if an algorithmic search terminates successfully, the interpretation is not necessarily possible without seeing the 'context' of the optimum, say, in a chart in which suboptimal regimes are presented as well. As for the presently considered WEC concept a combination of algorithmic and manual methods were applied successfully. By applying the method of dimensional analysis [19] we could not reduce the number of variables to be optimized, but, instead, by applying the so-called amplitude criterion of optimum [14] we could do just that; and, due to the simplicity of the model the response could be expressed explicitly, which greatly enhanced the efficiency of the numerics. The results of these procedures reveal that, indeed, the optimal solution is not unique, but long attenuatortype and wide terminator-type devices perform equally well. Furthermore, the dimensional analysis framework - if not for the envisaged purpose - could be utilized for the physical interpretation of the results.

The structure of the paper is as follows. In Sec. 2 a detailed mechanical model of the concept is given, including the equations of motion and the way irregular waves are accounted for. In Sec. 3 we review previous analysis of a free-floating box barge conducted in a nondimensional framework, and attempt the application of such methods to our WEC concept, which process sheds light on a possible method of optimization. In Sec. 4 we present results, and subsequently in Sec. 5 we discuss these results - providing some details of the optimization, and giving a physical interpretation of the results. In Sec. 6 we summarize our findings and draw conclusions.

\section{Mathematical model}

Mechanical model. The mechanical model of the box hull wave energy converter concept ${ }_{103}$ is shown in Fig. 1. Its details are explained as follows. The hull is a rectangular solid block, ${ }_{104}$ whose geometry is characterized by its breadth, draught, and length $(B, D, L)$. There are ${ }_{105}$ two active degrees of freedom: a translational one in the vertical direction, heave $(z)$, and ${ }_{106}$ a rotational one, pitch $(\varphi)$. The corresponding constraints are symbolized in the sketch 107 by a hinge, which is confined to moving in a vertical slide. In equilibrium the hinge always ${ }_{108}$ coincides with the still water level. This is a choice made in order to simplify the forthcoming ${ }_{109}$ analysis. The density of the hull is such that it is partially submerged, and the state of ${ }_{110}$ hydrostatic equilibrium is stable. The density, together with the geometry, determines the ${ }_{111}$ mass and inertia $(m, \theta)$. The elevation of the center of gravity (COG) is variable, but within ${ }_{112}$ limits of meeting the condition of hydrostatic stability. The effect of buoyancy is modeled ${ }_{113}$ mechanically by a linear spring characterized by a stiffness $s_{h}$, which links the mass with the ${ }_{114}$ 


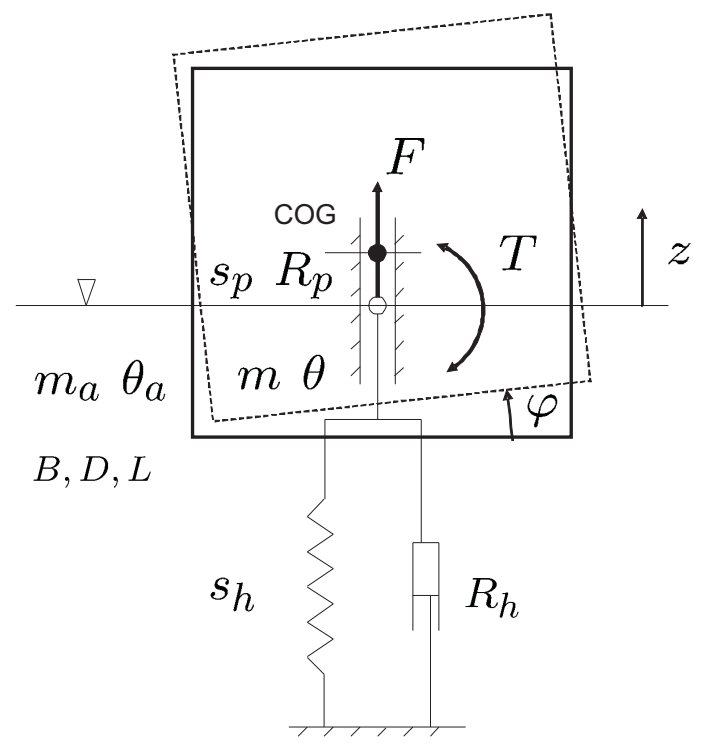

Figure 1: Mechanical model of the box-hull wave energy converter concept. The box barge, linked with the mechanical reference by dampers, oscillates in heave and pitch.

mechanical reference. In pitch, the spring is a torsional spring $\left(s_{p}\right.$, graphically not indicated in the sketch). In either DOF a damping effect is accounted for as well, characterized by damping coefficients $R_{h}$ and $R_{p}$, which are due to the radiation of waves when the float oscillates, entailing a loss of energy. A conjugate effect to this is that the moving body experiences an apparent increase of its mass and inertia as well $\left(m_{a}, \theta_{a}\right)$. This is commonly referred to as the added mass effect. The hydrodynamic damping and added mass effect are frequency-dependent $[20,15,21,14]$. PTO in either DOF is achieved by linear dampers $\left(k_{h}, k_{p}\right)$ connected in parallel with those that represent radiation damping (graphically not indicated in the sketch). That is, the PTO relies upon the mechanical reference. Due to wave action on the hull, there is a forcing of motion in the respective DOF's $(F, T)$. We shall use 2D, i.e., long-crested waves throughout this analysis, which travel along the length of the barge.

Governing equations and response. The equation of motion in the frequency domain can be written in a matrix form:

$$
\left[(i \omega)^{2}\left(\mathbf{M}_{\mathbf{0}}+\mathbf{M}_{\mathbf{a}}\right)+i \omega(\mathbf{K}+\mathbf{R})+\mathbf{S}\right] \mathbf{x}=\mathbf{Q},
$$

in which $\mathbf{x}=(z, \varphi)$ and $\mathbf{Q}=(F, T)$ are frequency dependent (phased) complex quantities; $i$ is the imaginary unit; $\mathbf{M}_{\mathbf{0}}$ and $\mathbf{K}$ are diagonal matrices of structural mass and (PTO) damping, respectively. This formulation is unchanged when considering all six DOF's of the rigid body, which is our starting point in the determination of $\mathbf{M}_{\mathbf{0}}$ and $\mathbf{K}$ as for the 2-DOF model. For the definition of the displacement, we consider the one point of the rigid body which is the intersection of its vertical center line and the axis of the hinge. The frequency-dependent radiation-diffraction parameters of eq. (1), the added mass $\left(\mathbf{M}_{\mathbf{a}}\right)$, radiation damping $(\mathbf{R})$, 
and force coefficients ( $\mathbf{Q}$, including Froud-Krylov and diffraction forces), are in fact not ${ }_{136}$ independent [14]. For our analysis they are obtained by using the ANSYS AQWA software ${ }_{137}$ package (AQWA-LINE suite), which implements a boundary element method algorithm [22]. 138

For the presently considered geometry and configuration, $\mathbf{M}_{\mathbf{a}}$ and $\mathbf{R}$ in 6 -DOF show the $\quad 139$ same pattern with regard to their nonzero entries $(\cdot)$ :

$$
\left[\begin{array}{llllll}
\cdot & 0 & 0 & 0 & \cdot & 0 \\
0 & \cdot & 0 & \cdot & 0 & 0 \\
0 & 0 & \cdot & 0 & 0 & 0 \\
0 & \cdot & 0 & \cdot & 0 & 0 \\
\cdot & 0 & 0 & 0 & \cdot & 0 \\
0 & 0 & 0 & 0 & 0 & \cdot
\end{array}\right]
$$

These matrices are also symmetric. On the other hand, the stiffness matrix $\mathbf{S}$ has diagonal ${ }_{141}$ nonzero entries only. These imply that heave is an independent DOF. Considering also that ${ }_{142}$ the hinge moves in a vertical slide, which can be modeled with infinite stiffness in sway, ${ }^{143}$ pitch is the only other active DOF remaining. Thus, the two active DOF's are independent. ${ }_{144}$ Consequently, the solution in either DOF can be given explicitly (with the example of heave) ${ }_{145}$ as follows:

$$
z=\frac{F}{-\omega^{2}\left(m+m_{a}\right)+i \omega\left(k_{h}+R_{h}\right)+s_{h}} .
$$

The force coefficient $f$ provided by AQWA is defined as the force for unit wave amplitude: ${ }_{147}$ $F=F_{e, 3}=f A$. With this, the mean power take-off, that is, the rate of energy conversion ${ }_{148}$ on the linear damper, is:

$$
P_{h}=k_{h}(\omega|z|)^{2} / 2
$$

and likewise in pitch.

In the independent DOF of heave the time-domain equation of motion assumes the ${ }_{151}$ following form:

$$
\begin{aligned}
& {\left[m+m_{a}(\infty)\right] \ddot{z}(t)+k_{h} \dot{z}(t)} \\
& +k_{r, h}(t) * \dot{z}(t)+s_{h} z(t) \\
& =f(t) * a(t) .
\end{aligned}
$$

This is a linear integro-differential equation, in which the asterisk $*$ denotes the operation $\quad 153$ of convolution; and the impulse response function is defined [14] as:

$$
k_{r, h}(t)=2 \mathcal{F}^{-1}\left[R_{h}(\omega)\right] .
$$

In the above, $\mathcal{F}^{-1}$ denotes the operation of (inverse) Fourier transformation. The frequency- ${ }_{155}$ dependent force vector $f(\omega)$ is similarly transformed into the time domain (but without a $2 \quad 156$ 
times multiplier); and for a regular wave the amplitude of water surface elevation in some reference position is:

$$
a(t)=A \sin (\omega t) .
$$

Time-domain equations for general 3D scenarios can be generated and solved using the AQWA-NAUT program suite. By doing so we can validate the above reconstructed 1-DOF frequency-domain equations of motion and subsequently obtained solutions. AQWA-NAUT recalculates the Froud-Krylov forces in every time step considering finite displacements and finite wave heights, i.e., nonlinear effects. Therefore, in order to validate linear equations, small wave heights have to be used. This is done in Appendix A.

Hydrostatic stability. Denoting the elevation of the COG by $z_{g}$ (positive upward), the structural inertia of a rectangular solid is calculated as:

$$
\theta=\frac{1}{12} m\left[L^{2}+4\left(D+z_{g}\right)^{2}\right]+m z_{g}^{2} ;
$$

and the stiffness in pitch is expressed as:

$$
s_{p}=s_{p 0}-m g z_{g} .
$$

In the latter $s_{p 0}$ is the stiffness if the COG coincides with the hinge. Notice that when $z_{g}$ is positive, the stiffness is decreasing according to an inverted pendulum effect. On the other hand, the inertia is always greater for a nonzero $z_{g}$. The stiffness and the inertia determines the natural frequency (squared):

$$
\alpha_{p}^{2}=\frac{s_{p}}{\left(\theta+\theta_{a}\right)} .
$$

For some values of $z_{g}$ the box can capsize, however, and its upper and lower critical values need to be identified.

The following formula provides a connection between the elevation of COG and the density of float:

$$
z_{g}=D\left(\frac{\rho_{w}}{2 \rho}-1\right) .
$$

For hydrostatic stability in pitch it is required that the metacentric height be positive [23]:

$$
G M=B M-B G>0,
$$

where

$$
B M=I / V .
$$

In the above, the metacenter $(M)$ and the elevation of the COG $(G)$ are given relative to the point of attack of the integrated buoyancy force $\left(z_{B}=-D / 2\right) ; I$ is the second moment 
of inertia of the water plane area with respect to the $x$-axis; and $V=B D L$ is the displaced ${ }_{180}$ (water) volume. In terms of the design parameters, criterion (11) takes the following form: ${ }_{181}$

$$
z_{g}<\frac{(L / 2)^{2}}{3 D}-\frac{D}{2}
$$

which provides an upper bound on $z_{g}$. A lower bound is determined by the condition of ${ }_{182}$ preventing complete immersion:

$$
-D / 2<z_{g}
$$

which is equivalent to requiring $\rho<\rho_{w}$.

Wave spectrum. Up to this point we have considered regular waves only. As we detail 185 it in the next section, regular waves do not in fact yield an optimum when using the lin- ${ }_{186}$ ear model, and the resonance effect is also not 'robust' when considering irregular waves. ${ }^{187}$ Since in reality sea waves are never regular, already in this preliminary analysis we consider $\quad{ }_{188}$ irregular waves of some finite bandwidth. For our purposes it will suffice to use a simple 189 symmetric model spectrum, $S(f)(\omega=2 \pi f)$, which is referred to as the water surface el- ${ }_{190}$ evation spectrum. We define this spectrum using the functional form of the probability 191 density of the standard normal distribution of mean $\mu$ and variance $\sigma^{2}$ (with no physical ${ }_{192}$ meaning intended): $\phi(x)=\frac{1}{\sqrt{2 \pi \sigma^{2}}} \exp \left[-(x-\mu)^{2} /\left(2 \sigma^{2}\right)\right]$. It is a suitable choice given that 193 it is normalized so that $\int_{-\infty}^{\infty} \phi(x) d x=1$. In order to specify $S$, we define the significant 194 wave height $H_{s}$ in terms of the zeroth moment of the spectrum, $m_{0}=\int_{0}^{\infty} S(f) d f$, such as: ${ }_{195}$ $H_{s}=4 \sqrt{m_{0}}$ [24]. We note that since the lower boundary of the integral for $m_{0}$ is not $-\infty{ }_{196}$ but 0 , there is a small error using $\phi(x)$. Collecting the formulas above, the wave spectrum ${ }_{197}$ is written in full as:

$$
\begin{aligned}
& S(f)=\left(\frac{H_{s}}{4}\right)^{2} \times \\
& \times \frac{1}{\sqrt{2 \pi \sigma^{2}}} \exp \left[-\left(f-f_{0}\right)^{2} /\left(2 \sigma^{2}\right)\right],
\end{aligned}
$$

in which $f_{0}\left[\mathrm{~s}^{-1}\right]$ is the peak frequency; and $\sigma\left[\mathrm{s}^{-1}\right]$ can be regarded as a bandwidth parameter. In our analysis we use narrowband waves $(\sigma=0.05)$ which grant us the benefit of having a physical meaning of the results in most of the considered parameter regime; whereas using more realistic broadband spectra, like e.g. Pierson-Moskowitz or JONSWAP, would render the physical meaning in some regimes.

Mean power take-off. In frequency domain the power spectrum of an actual response can be obtained simply from the synthesis of the power spectrum corresponding to (over the frequency range uniformly) unit wave amplitudes, $P_{1}=P_{h}(A=1)+P_{p}(A=1)\left[\mathrm{Wm}^{-2}\right]$ using e.g. eqs. (2) and (3), and the actual water surface elevation spectrum, $S(f)\left[\mathrm{m}^{2} \mathrm{~s}\right]$, $[25]$ :

$$
P(f)=2 P_{1}(f) S(f) ;
$$


from which the overall mean PTO is:

$$
\bar{P}=\int_{0}^{\infty} P(f) d f .
$$

In numerical calculations the power (3) and wave spectra (15) are discretized $\left[S_{i}=S\left(f_{i}\right)\right]$; and thus the integral (17) is replaced by a sum, the number of terms in which are limited by a (reasonably chosen) maximal cut-off frequency.

\section{Rationale and methods of analysis}

Free-floating box barge. Prior to the main analysis in this paper we attempted to reconstruct Kraemer's results [3] regarding a free-floating box barge, lacking any power take-off mechanism or external damping. The methodology and parameters of our reconstruction are as follows. The box barge is assumed to be halfway submerged. Furthermore, we fixed $L=20$ and $V=B D L=640$, and varied $B$ and $D$ jointly, and varied $\omega$ as well. The frequency $\omega$ is sampled fine enough so that very narrow-banded resonance peaks could be resolved. Sample values for $D$, which would imply values for $B$, are chosen such as: $2 D=4$, $6,8,10,10.8,11.2,12,12.5$. The fixed regular wave height is: $H=0.1$. Note that $H=2 A$. The reconstructed results are displayed in Fig. 2. The oscillation amplitude in pitch as a function of the frequency ratio $\left(\pi_{1}=\omega / \alpha_{p}\right)$ and the wavelength ratio $\left(\pi_{4}=\lambda / L\right)$ is plotted. In the latter the wavelength of a regular wave of frequency $\omega$ in deep water is:

$$
\lambda=2 \pi g / \omega^{2} .
$$

The independent variables $\pi_{1}$ and $\pi_{4}$ are nondimensional, which are varied via dimensional ones, $B$ and $\omega$. It can be shown that the considered problem can be described in terms of either four independent dimensional or four nondimensional variables. (We will detail such an analysis shortly as for the complete WEC model described in Sec. 2.) The remaining two nondimensional variables - held constant in Fig. 2 - can be defined as $\pi_{3}=V / H^{3}$, the nondimensionalized volume, and $\pi_{2}=B D / H^{2}$, a constraint on the aspect ratios. Results are reconstructed using a linear frequency-domain model [Fig. 2 (a)], as well as a nonlinear timedomain model [Fig. 2 (b)]. Notice the different physical units for the oscillation amplitude associated with the two diagrams, and also that in panel (a) the range of the frequency ratio $\omega / \alpha_{p}$ shown is much shorter in order to resolve very narrow-banded resonance peaks.

The fact that the linear model deviates greatly from the nonlinear model for larger wavelength ratios indicates that the former is not valid. The oscillation amplitudes that can be read off of the diagram in Fig. 2 (a) are certainly not realistic. However, for small oscillation amplitudes and wave heights the two models agree well, as demonstrated in Appendix A (in case of the externally damped structure, to be introduced shortly below). The diagram in Fig. 2 (b) corresponding to the nonlinear model reveals a pair of optimal frequency ratio and wavelength ratio:

$$
\pi_{1}=\omega / \alpha_{p} \approx 1 \text { and } \pi_{4}=\lambda / L \approx 5
$$




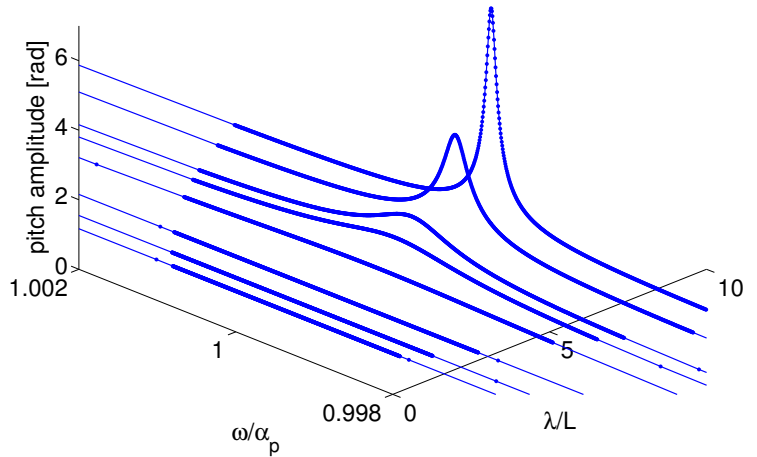

(b)

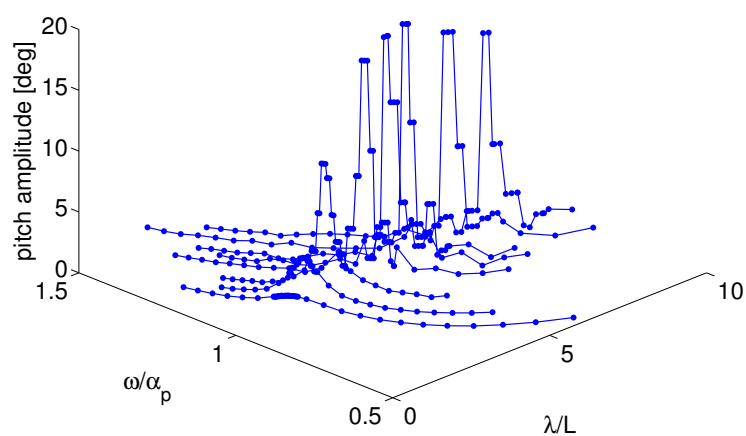

Figure 2: Oscillation amplitude of a free-floating box barge in pitch as a function of the frequency ratio $\left(\pi_{1}\right)$ and the wavelength ratio $\left(\pi_{4}\right)$, using (a) a frequency-domain and (b) a time-domain model. Sampling of the response is indicated by markers. In both cases we fixed $L=20$ and $V=640$; and a series of draught values were set, such as: $2 D=4,6,8,10,10.8,11.2,12,12.5$. The wave height in both cases is: $H=0.1$. Notice the different physical units as for the oscillation amplitude. 
Such an optimum is due to the progressively increasing, i.e., nonlinear, restoring buoyancy force characteristic deriving from the hull shape. With regard to the wave frequency, on the other hand, the optimum corresponds to resonance - as expected from the theory of WECs [14]. The linear model does not in fact admit an optimal wavelength ratio, but it yields ever-increasing oscillation amplitude and decreasing response bandwidth. This effect is discussed in more detail in Appendix B. The oscillation amplitude is in fact very closely related to the inverse of the bandwidth, and they are progressively increasing. This is a feature of our results based on the linear model which disagree - for a yet unidentified reason - with the results reported by Kreamer based, apparently, also on a linear model: Kraemer reported a degressive increase and a seemingly unaffected bandwidth, with no indication of the behavior beyond the range of wavelength ratios examined (0-5). Apart from this, based on our results we conclude that a linear model in the present setting is not suitable to predict the optimum, as it is outwith the range of model validity.

Power take-off by dampers. As a step further, the model described in Sec. 2 can be obtained by linking the box hull to the mechanical reference by dampers. Next, the framework of analysis used in case of the free-floating box barge is applied to the WEC with the dampers. Now the objective function of optimization is the power. The objective of this exercise is to see if the conditions for optimum (19) hold. The following setup is picked for this analysis. The barge is assumed to be halfway submerged, that is, its COG lies on the axis of hinge. The fixed size of the barge is: $\rho_{w} V=2 \cdot 10^{5} \mathrm{~kg}$. The breadth is set to be $B=5$, and a series of draughts are considered, such as: $D=2.2,2.4,2.6,2.8,3,3.1,3.2$, $3.25,3.3,3.35,3.4,3.425,3.45,3.475,3.5,3.525$. With this, the definition of $\pi_{2}=B D / H^{2}$ is modified to $\pi_{2}=L D / H^{2}$. However, this makes no difference in the interpretation. Using a linear model, whose results are shown in Fig. 3 (a), a condition for optimum in terms of $\lambda / L$ does not exist, the amplitude is increasing and the bandwidth is decreasing monotonically - very much alike the displacement response of the free floating barge.

Introducing irregular waves [Fig. 3 (b)], with a nonzero bandwidth of the wave spectrum, an optimal value of $\lambda / L$ emerges, which is about 2 . It is significantly smaller than 5 under (19), owing to the larger bandwidths of the response at smaller values of the wavelength ratio. That is, the conditions for optimum (19) do not hold. We conclude thus that besides nonlinear effects, irregular waves are also found to be able to render very high amplitude responses and to create an optimum. Furthermore, the diffraction forces are more significant under the conditions of optimal performance in irregular seas in comparison with the conditions necessary for the most intensive displacement response of the free-floating barge exposed to regular waves.

A further important conclusion to draw is that with finite bandwidth irregular waves the response stays in the linear regime, and so we can make the favorable choice of using the linear frequency-domain model for our optimization procedure, which requires much less numerical effort when calculating the response in comparison with the use of the (nonlinear) time-domain model.

Dimensional analysis. In order to have a direction for pursuing optimization, a complete dimensional analysis of the WEC is given next. It is outlined in Table 1. According to the standard procedure [19] on the left hand side of the table listed are all the dimensional 
(a)
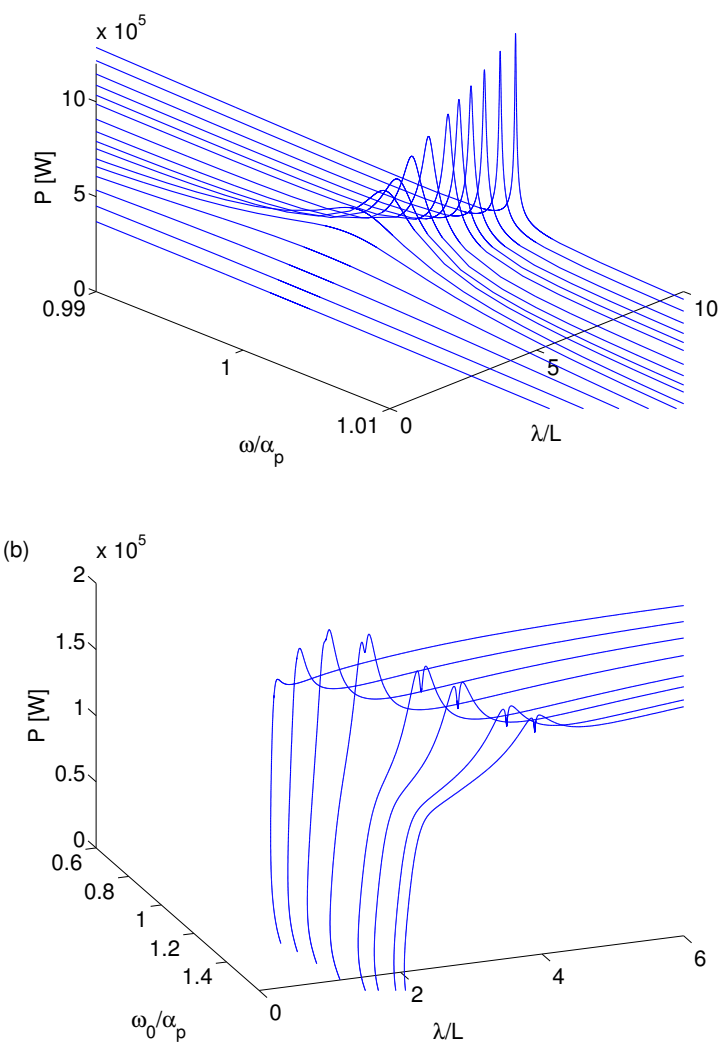

Figure 3: (a) The total power take-off (PTO) of a box barge as a function of the frequency ratio $\left(\pi_{1}\right)$ and the wavelength ratio $\left(\pi_{4}\right)$. We fixed $\rho_{w} V=2 \cdot 10^{5} \mathrm{~kg}$ and $B=5$; and a series of draught values are considered such as: $D=2.2,2.4,2.6,2.8,3,3.1,3.2,3.25,3.3,3.35,3.4,3.425,3.45,3.475,3.5,3.525$. (b) The total PTO (with the first few sample values of $D$ as listed above) achieved when using irregular waves. (Instead of $\omega$ we write $\omega_{0}$, the peak frequency of the frequency spectrum.) 
Table 1: Outline of dimensional analysis

\begin{tabular}{|l|l|l|}
\hline Dimensional variables & Physical unit & Nondimensional variables \\
\hline$\omega_{0}$, peak frequency & $(\mathrm{rad} \cdot) \mathrm{s}^{-1}$ & $\pi_{1}=\omega_{0} / \alpha_{p}$ \\
$B$, breadth & $\mathrm{m}$ & $\pi_{2}=\omega_{0} / \alpha_{h}$ \\
$D$, draught & $\mathrm{m}$ & $\pi_{3}=B D L / H_{s}^{3}$ \\
$L$, length & $\mathrm{m}$ & $\pi_{4}=\lambda_{0} / L$ \\
$\rho$, density of barge & $\mathrm{kg} \cdot \mathrm{m}^{-3}$ & $\pi_{5}=\rho / \rho_{w}$ \\
$k_{h}$, damping in heave & $\mathrm{kg} \cdot \mathrm{s}^{-1}$ & $\pi_{6}=k_{h} /\left|Z_{i, h}\left(\omega_{0}\right)\right|$ \\
$k_{p}$, torsional $*$ in pitch & $\mathrm{kg} \cdot \mathrm{m}^{2} \cdot\left(\mathrm{rad}^{-1} \cdot\right) \mathrm{s}^{-1}$ & $\pi_{7}=k_{p} /\left|Z_{i, p}\left(\omega_{0}\right)\right|$ \\
\hline$\rho_{w}$, water density & $\mathrm{kg} \cdot \mathrm{m}^{-3}$ & \\
$g$, gravitational acceleration & $\mathrm{m} \cdot \mathrm{s}^{-2}$ & \\
$H_{s}$, significant wave height & $\mathrm{m}$ & \\
\hline$P$, power & $\mathrm{kg} \cdot \mathrm{m}^{2} \cdot \mathrm{s}^{-3}$ & $\pi_{0}=P / P_{r e f}$ \\
\hline
\end{tabular}

Table 2: Account of dimensional analysis

\begin{tabular}{|l|l|}
\hline Number of $p$ 's & $n=10$ \\
Number of fixed $p$ 's & $n_{F}=3$ \\
Number of dimensionally independent $p$ 's & $k=3$ \\
Number of dimensionally independent fixed $p$ 's & $k_{F}=3$ \\
\hline
\end{tabular}

parameters $(p$ 's) that determine the objective function, the power $P$. These include design parameters, $B, D, L, \rho, k_{h}, k_{p}$, environmental parameters, $f_{0}, H_{s}$, and also global parameters, $\rho_{w}=1024.4 \mathrm{~kg} / \mathrm{m}^{3}, g=9.807 \mathrm{~m} / \mathrm{s}^{2}$. The latter three are fixed in this analysis. All these parameters together constitute a complete set, i.e., they uniquely define the problem (e.g. the way we need to do so for computations using ANSYS AQWA) and the solution. Applying Buckingham's $\pi$ theorem [19], modified for the case when some $p$ 's are fixed, the number of independent $\pi$ 's that fully describe the problem is:

$$
N=(n-k)-\left(n_{F}-k_{F}\right)=7 .
$$

which is not fewer than the $\left(n-n_{F}\right)$ dimensional variables. For an account of the dimensional analysis see Table 2 . Thus, the dimensional analysis could not reduce the dimensionality or order of the problem, and so optimization cannot be simplified in this way. On the right hand side of Table 1, accordingly, we list a complete set of nondimensional variables $(\pi$ 's). In there $P_{r e f}$ is an arbitrary reference power level; and the intrinsic impedance (with the example of heave) is defined as:

$$
Z_{i, h}=i \omega\left(m+m_{a}\right)+R_{h}+s_{h} /(i \omega) .
$$

The $\pi$ 's are defined not in a conventional way but so that they can provide a physical meaning of an optimal solution, and possibly facilitate the application of some criteria of optimum, based on theory or perhaps experience. This could open up another way of utilizing dimensional analysis in order to reduce the dimensionality of the problem. It is 
straightforward to show that in case of a 1-DOF (2D or 3D) linear oscillatory system maximal 302 PTO is achieved by setting:

$$
k=\left|Z_{i}\right|
$$

at any ${ }^{1}$ frequency. This setting is to satisfy the amplitude criterion of optimum. In our 304 case it dictates that $\pi_{6}=1$ ! and $\pi_{7}=1$ ! (Note that we denote by the exclamation ${ }_{305}$ mark a 'requirement' for a certain objective (optimum here), as in variational calculus. 306 The exclamation does not denote here the factorial of a nonnegative integer.) With 1- 307 DOF a complementary criterion for optimum is the phase criterion, which requires that 308 the excitation force (the sum of Froude-Krylov and diffraction forces) be in phase with the 309 velocity of oscillation, which is in fact satisfied in resonance [14]. One might think that in 310 our case the phase criterion dictates that either $\pi_{1}=1$ ! or $\pi_{2}=1$ ! However, it makes no 311 immediate contradiction proposing that the optimum might poses itself as a 'compromise', 312 in which case there is no perfect resonance in any of the DOF's; or in other words: one ${ }_{313}$ can assume that the optimum occurs when $\pi_{1} \neq 1$ and $\pi_{2} \neq 1$ simultaneously. Certainly, 314 resonance in both DOF's in the same time can be satisfied ${ }^{2}$ only in special cases. Since in 315 case of our 2-DOF and 3D WEC there are other variables too that determine optimum, it 316 is unlikely that the ultimate optimum would occur in that special situation. One of the ${ }_{317}$ further variables is the device size, $\pi_{3}$, which we will fix ${ }^{3}$. Another one is the wavelength 318 ratio, for which - as demonstrated above - we do not have a universally applicable rule of ${ }_{319}$ optimal setting. There is also no trivially optimal setting as for the density ratio $\pi_{5}$.

Customized framework of optimization. With the application of some criteria of opti- 321 mum, and also directly fixing the size $\pi_{3}$, the dimensionality of the problem could be reduced 322 in principle. In practice, however, no advance has been made, because the mapping between ${ }_{323}$ the $p$ 's and $\pi$ 's involves the computationally expensive diffraction analysis. Therefore, our ${ }_{324}$ approach is to pursue optimization in terms of the dimensional parameters, and then trans- ${ }_{325}$ late the results into terms of the nondimensional ones for the purpose of interpretation, in 326 order to see, for example, if optimum entails resonance. 327

In general the optimization is increasingly more complicated with the increasing number 328 of variables. In our case, however, owing to the simplicity of the mathematical model and the ${ }_{329}$ applicability of theory, the procedure of optimization can be broken down into subsequent 330 manageable stages as follows.

(i) As our starting point, in accordance with the first column of Table 1 we write the objec- ${ }_{332}$ tive function, the power take-off, as a function of the dimensional design parameters 333 and the one environmental parameter, the (peak) frequency, with respect to which 334 there is a nontrivial optimum:

$$
P_{(\mathrm{i})}\left(\omega_{0}, B, D, L, \rho, k_{h}, k_{p}\right) \text {. }
$$

\section{5}

\footnotetext{
${ }^{1}$ The amplitude criterion is usually formulated assuming that resonance takes place already $[k=R(\omega=$ $\alpha)$ !], which is a special case of eq. (22).

${ }^{2}$ With regular waves in a $2 \mathrm{D}$ setting, this is the condition for the total destruction or stopping of the incident waves, which results in $100 \%$ efficiency.

${ }^{3}$ For finding an economic optimum, the size that determines costs has to be varied subsequently.
} 
We can separate the parameters into two groups by a semicolon such that on its left we write the variables with respect to which we are looking for the optimum, and on its right we write the variables whose value we fix.

(ii) Although we are interested in finding an optimum with respect to $B$ and $D$, we will search for this optimum - or, give a representation of the dependence of $P$ on them visually, and so we write $B$ and $D$ immediately right to the semicolon: $P_{(\mathrm{ii})}\left(\omega_{0}, L, \rho, k_{h}, k_{p} ; B, D\right)$.

In order to eliminate variables from the left of the semicolon,

(iii) first the size $V=B D L$ is fixed, which - having fixed $B$ and $D$ - amounts to fixing $L$, and so we write:

$P_{(\mathrm{iii})}\left(\omega_{0}, \rho, k_{h}, k_{p} ; B, D, V\right)$.

So given a fixed geometry, the diffraction analysis can be carried out for a range of frequencies $\omega$.

(iv) The density $\rho$ can be varied via varying $z_{g}$ [more precisely $\rho=\rho\left(z_{g}, D\right)$; see eq. (10)], which takes its effect through the analytically given response [see eqs. (2), (3), (7), (8), (9)]. $P_{\text {(iv) }}\left(\omega_{0}, z_{g}, k_{h}, k_{p} ; B, D, V\right)$

(v) The damping coefficients are set according to eq. (22) in a straightforward manner to produce $P_{1}$, but substituting $\omega_{0}$ for $\omega$ in that equation before applying eq. (16): $k_{h / p}=k_{h / p}\left(\omega_{0}, B, D, V, \rho\right)$. $P_{(\mathrm{v})}\left(\omega_{0}, z_{g} ; B, D, V\right)$

(vi) Finally the optimum with respect to $\omega_{0}$ and $z_{g}$ can be found by a nested procedure: we solve the equation $P_{(\mathrm{v}), z_{g}}^{\prime}\left(\omega_{0}\left(z_{g}\right), z_{g} ; B, D, V\right)=0$ in which $\omega_{0}\left(z_{g}\right)$ is found by solving $P_{(\mathrm{v}), \omega_{0}}^{\prime}\left(\omega_{0} ; z_{g}, B, D, V\right)=0$

Regarding $P_{(\mathrm{v})}\left(\omega_{0} ; z_{g}, B, D, V\right)$ involved in the latter, refer to Fig. 11 (b) for a visual clue, where slices of the analytically given function $P_{(\mathrm{v})}\left(\omega_{0}, z_{g} ; \cdot\right)$ at various fixed values of $z_{g}$ can be seen.

The feasibility of our method is due in large part to the fact that we have to look for the maximum of functions of a single variable at a time. We achieved this by using Matlab's function fmincon, which implements a constrained optimum search algorithm. This way the optimal power (with fixed device size) can be mapped out as a function of $B$ and $D$ to yield a chart, which is easy to represent and interpret, and facilitates the ultimate purpose of indicating the optimal geometry.

We note that in order to nondimensionalize the objective function $P$, we used an arbitrary reference power level $P_{r e f}$. A more meaningful way to nondimensionalize $P$ would be dividing by the power in the incident waves in a window which is equal with the width of the WEC, $P_{w}[26]$. This nondimensional quantity is called the relative capture width: 


$$
\mathrm{RCW}=P / P_{w},
$$

in which

$$
P_{w}=\frac{\rho_{w} g^{2}}{64 \pi} H_{s}^{2} T_{e} B
$$

This quantity is a mean value. In the definition $T_{e}$ is the energy period, which will be 376 approximated as $2 \pi / \omega_{0}$, because the wave spectrum to be used is narrow-banded. In general, 377 however, the energy period of an irregular wave is different from its zero mean crossing 378 period $\left(T_{z}\right): T_{e}=m_{-1} / m_{0}$ and $T_{z}=m_{0} / m_{2}$ [26]. $T_{e}$ can be interpreted as the wave period 379 of a regular wave (of equal significant wave height $H_{s}=\sqrt{2} H$ ) which carries the same 380 energy as the irregular wave in question. We note that for the spectrum defined above 381 (15) $m_{-1}$ cannot be obtained analytically. Although more meaningful, the RCW is not so 382 useful as the objective function of optimization, because it is the power itself which has to be ${ }_{383}$ maximal, which does not necessarily occur under the same conditions as the maximal RCW. 384 Nevertheless, we will provide a chart of the RCW too, because it can indicate if the results 385 are realistic/correct or not. For example, for a wide $(B \gg D, L)$ terminator-type device, 386 exposed to irregular waves, $\mathrm{RCW}<1$ can only be realistic, even under optimal conditions. 387 As for a long $(L \gg B, D)$ attenuator-type device, $\mathrm{RCW} \gg 1$ is possible, while it would not ${ }_{388}$ necessarily outperform a terminator-type device of the same size $(V)$.

\section{Results}

In this section we present optimal figures for the objective function of optimization, 391 the power take-off $P$, and of the variables $\omega_{0}$ and $z_{g}$, i.e., the dependent and independent 392 variables of $P_{(\mathrm{v})}\left(\omega_{0}, z_{g} ; \cdot\right)$. Optimal figures for some derived variables defined previously are ${ }_{393}$ also shown. The results are presented in terms of color charts showing the various quantities 394 mapped out in terms of the geometric parameters $B$ and $D$. In terms of point (iii) of the 395 previous section the mass of device is fixed to be: $\pi_{3} H_{s}^{3} \rho_{w}=2 \cdot 10^{5} \mathrm{~kg}$. Lower bounds on the 396 geometric parameters are imposed such as: $B_{\min }=2, D_{\min }=1, L_{\min }=2$. An upper bound 397 on $D$ is independently imposed $\left(D_{\max }=10\right)$, because of uninteresting features for larger 398 values of it. Within their respective ranges, $B$ and $D$ are sampled with increments of 2 and 399 $0.2 \mathrm{~m}$, respectively, and the cartesian product of the resulting sets of sample values is taken 400 to sample the $B-D$ plane. Sample values which do not satisfy that $L>L_{\min }$ are ignored; i.e., ${ }^{401}$ the considered domain of the $B-D$ plane is bounded by the graph of a reciprocal function. ${ }_{402}$ For all relevant computations whose results are presented in this section the significant wave ${ }_{403}$ height is fixed to be $H_{s}=2 \sqrt{2}$. This is to establish equivalence in an approximate sense with ${ }_{404}$ a regular wave of unit amplitude, considering that rather narrow-banded irregular waves are ${ }_{405}$ used $(\sigma=0.05)$.

In Fig. 4 color charts of power in heave (c), (d), pitch (e), (f), and their sum (a), (b), 407 are displayed. Color bars are included to indicate the magnitude of power for the various ${ }_{408}$ $(B, D)$ scenarios. For certain scenarios we have coexisting local optima. Diagrams on the ${ }_{409}$ left (right) correspond to results when the optima are due to resonance in pitch (heave). ${ }_{410}$ 

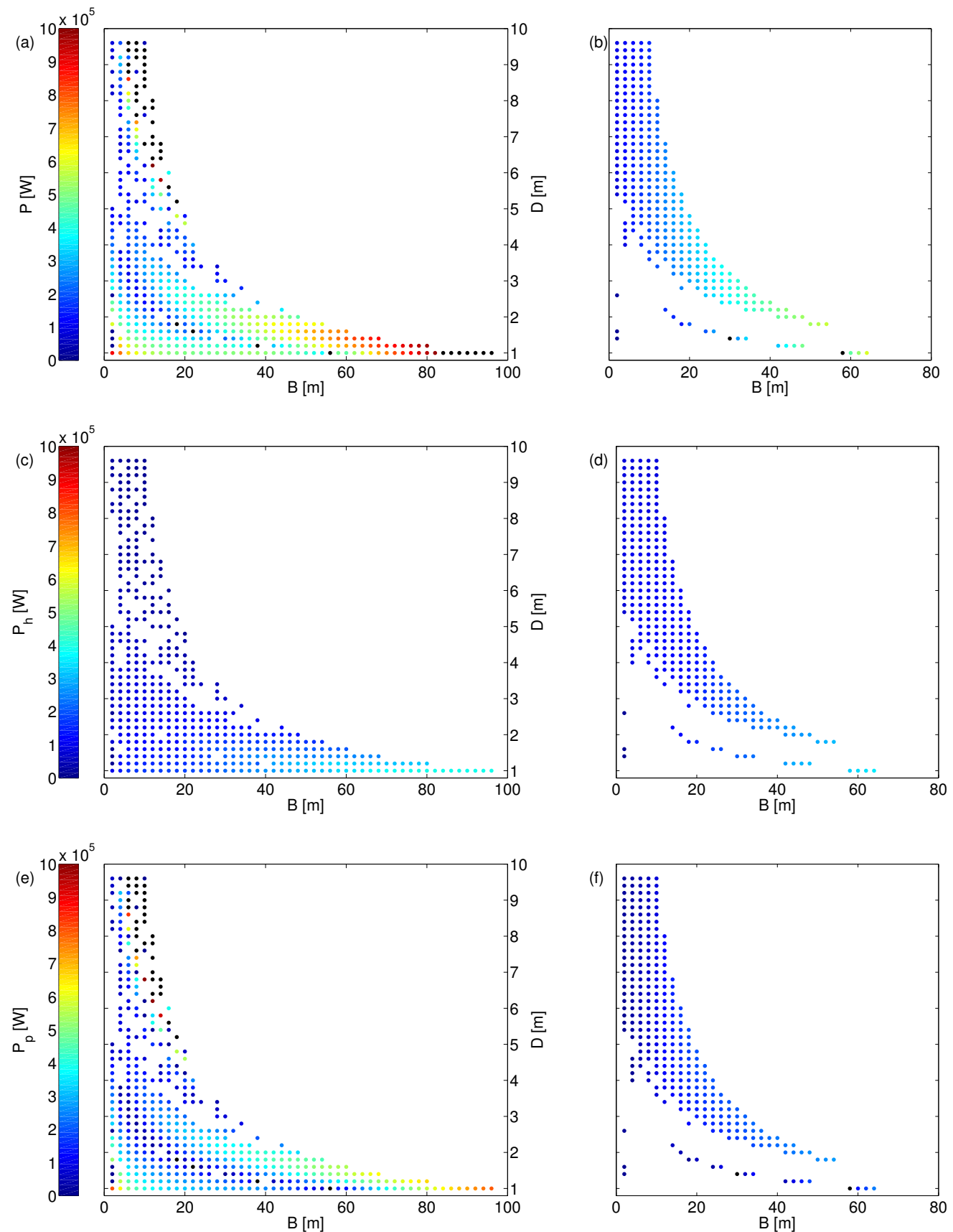

Figure 4: Power take-off in pitch (e), (f), heave (c), (d), and the sum of these (a), (b), depending on geometric parameters of the box hull, the breadth $B$ and draught $D$. Diagrams on the left (right) [e.g. (a) $((b))$ ] correspond to results when optima are due to resonance in pitch (heave). A significant wave height of $H_{s}=2 \sqrt{2}$ was used. For other settings for the simulations, refer to the main text. (An overbar is not used here to denote mean power in Watts.) 

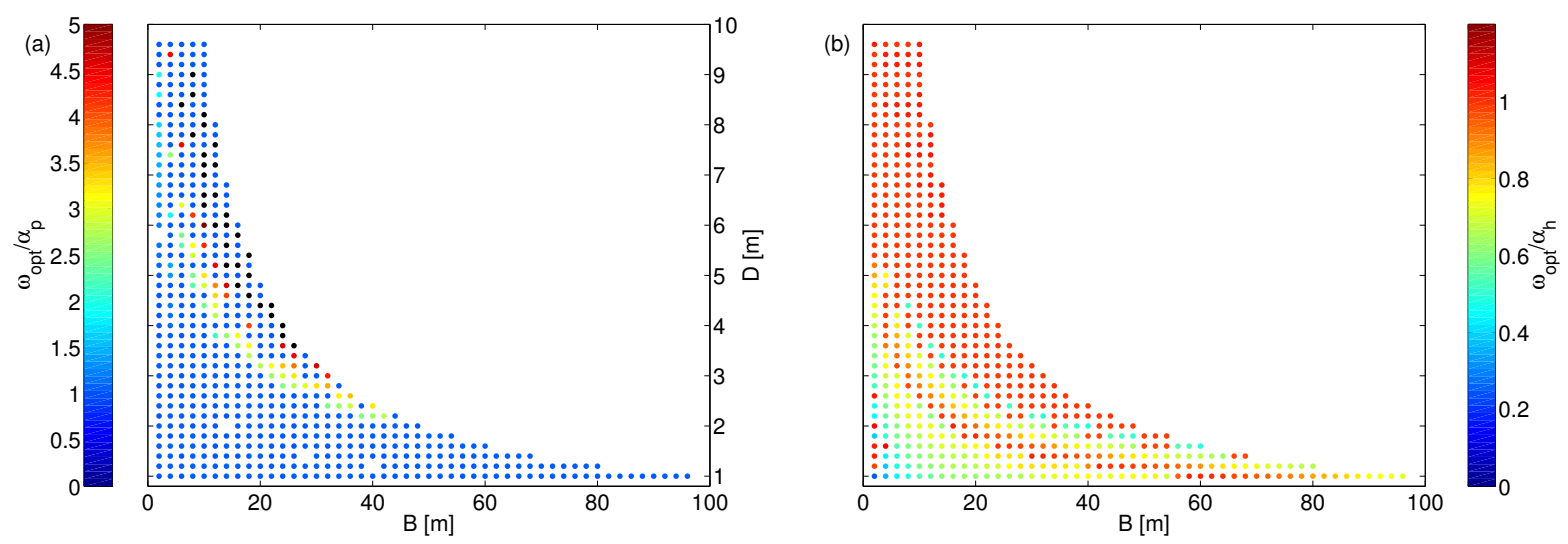

Figure 5: Frequency ratio corresponding to data that Fig. 4 was produced from.

Figure 5 indicates whether the optimum (the better performing one when there are two local ${ }_{411}$ optima) is due to a resonance in pitch or heave with the appropriate frequency ratio being ${ }_{412}$ nearly unity. For the construction of this figure natural frequencies were calculated using ${ }_{413}$ eq. (9), and optimal (peak) frequencies (shown in Fig. 6), along with optimal $z_{g}$ 's (shown in ${ }_{414}$ Fig. 7) (or optimal $\pi_{5}^{\prime} s$ ), were found using Matlab function fmincon (as described in point ${ }_{415}$ (vi) in the previous section). Two initial guess values (IV) were set such as: $\omega_{0}=\alpha_{p}$ and ${ }_{416}$ $\omega_{0}=\alpha_{h}$, with $z_{g}=z_{\text {min }}$ in both cases. With this setting, when there is only one optimum, ${ }_{417}$ i.e., when a single resonance peak exists, the solution converges to the unique optimum using ${ }_{418}$ either IV. Regarding the subsequent optimum search for $z_{g}$, since the surface that represents ${ }_{419}$ the power take-off over the plane of $\omega_{0}-z_{g}$ features a ridge (which ridge represents resonance; 420 see Fig. $11(\mathrm{~b}))$, the arbitrary choice for the IV's $z_{g}=z_{\min }$ should suffice. Note that in ${ }_{421}$ order to ensure the accuracy of calculating the power through eqs. (16) and (17), $\omega$ has to ${ }_{422}$ be sampled sufficiently for the purpose of resolving even extremely narrow-banded responses ${ }_{423}$ due to resonance.

For all the color charts in this section (except those of $z_{\text {min,max }}$ and $\omega_{\text {opt }} / \alpha_{p}(h)$ ) some data 425 points are missing, because either fmincon did not terminate successfully or the correctness ${ }_{426}$ of data was dubious. The condition to filter them out was set as follows:

$$
\left|\omega_{\text {opt }} / \alpha_{p(h)}-1\right|<0.03 \text {. }
$$

As mentioned above, it can happen that there are two distinct (coexisting) peaks of resonance ${ }_{428}$ in pitch and heave. In this case the charts would overlap, and so we chose not to present ${ }_{429}$ them in one diagram, but rather separately: the chart corresponding to resonance in pitch ${ }_{430}$ (heave) on the left (right). Charts of the wavelength ratio $\left(\pi_{4}\right)$ and RCW are displayed in ${ }_{431}$ Fig. 8 and Fig. 9, respectively. In Fig. 7 and elsewhere black markers are used when the 432 value is greater than the subjectively chosen upper limit to be indicated by colors (see the ${ }_{433}$ color bars). This approach was taken for the reason that 'outliers' did not carry valuable ${ }_{434}$ information, and their inclusion in the range of the color bar would result in poor visual ${ }_{435}$ 

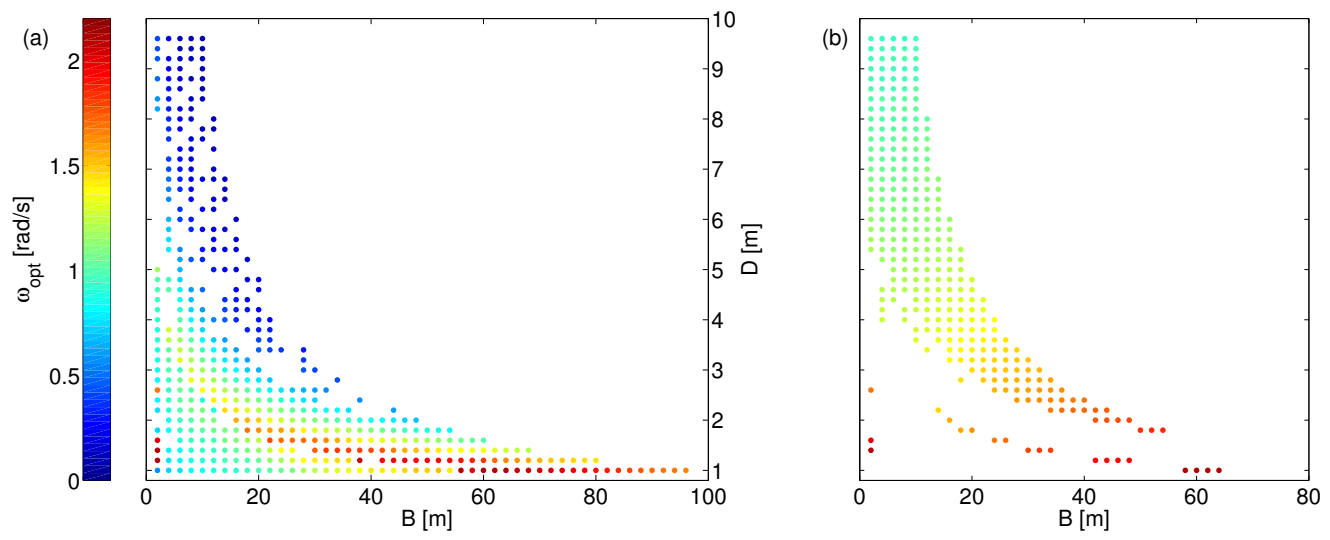

Figure 6: Optimal (peak) frequency corresponding to data that Fig. 4 was produced from.
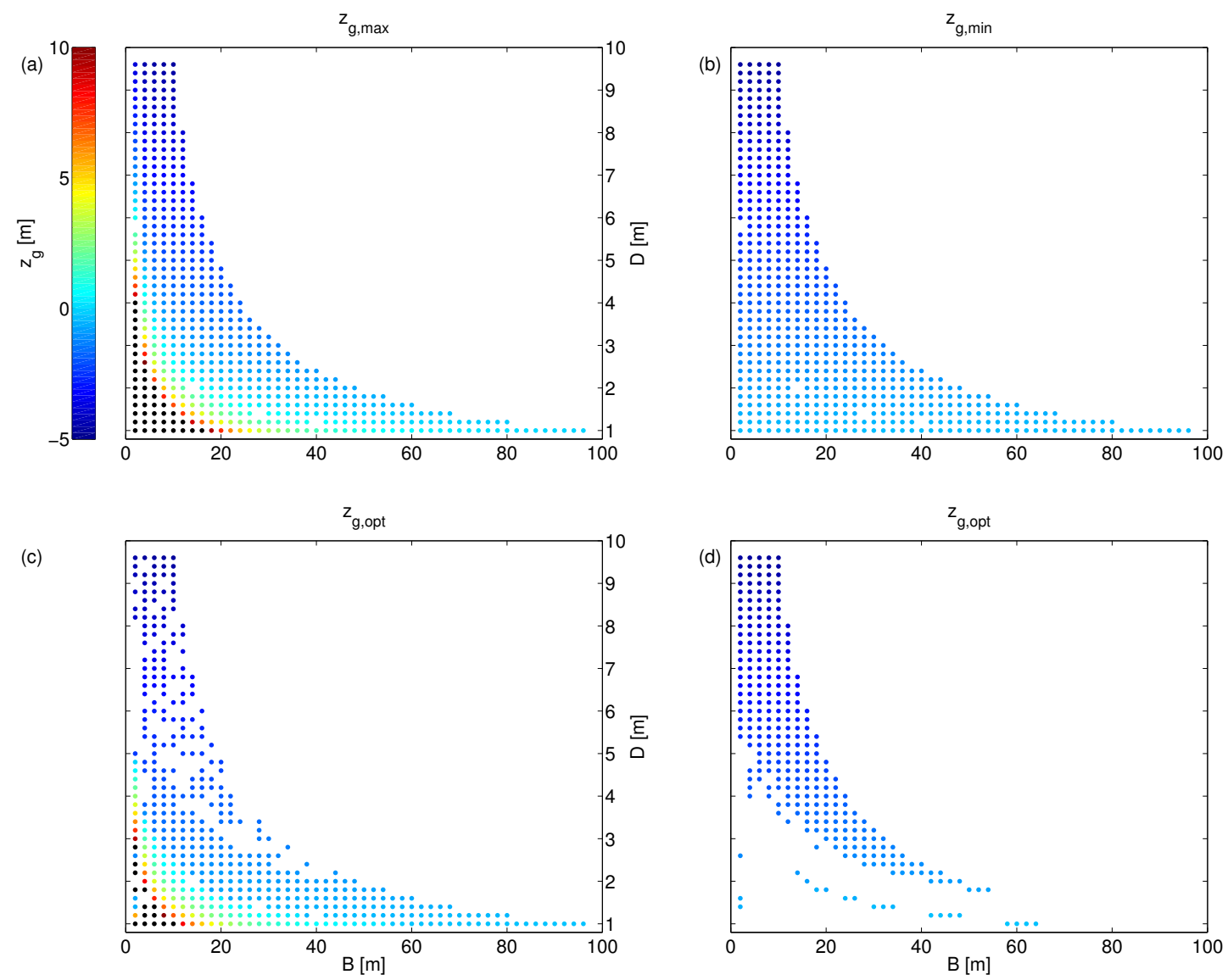

Figure 7: Upper (a) and lower (b) boundaries for the elevation of the COG, and its optimal values (c), (d). 

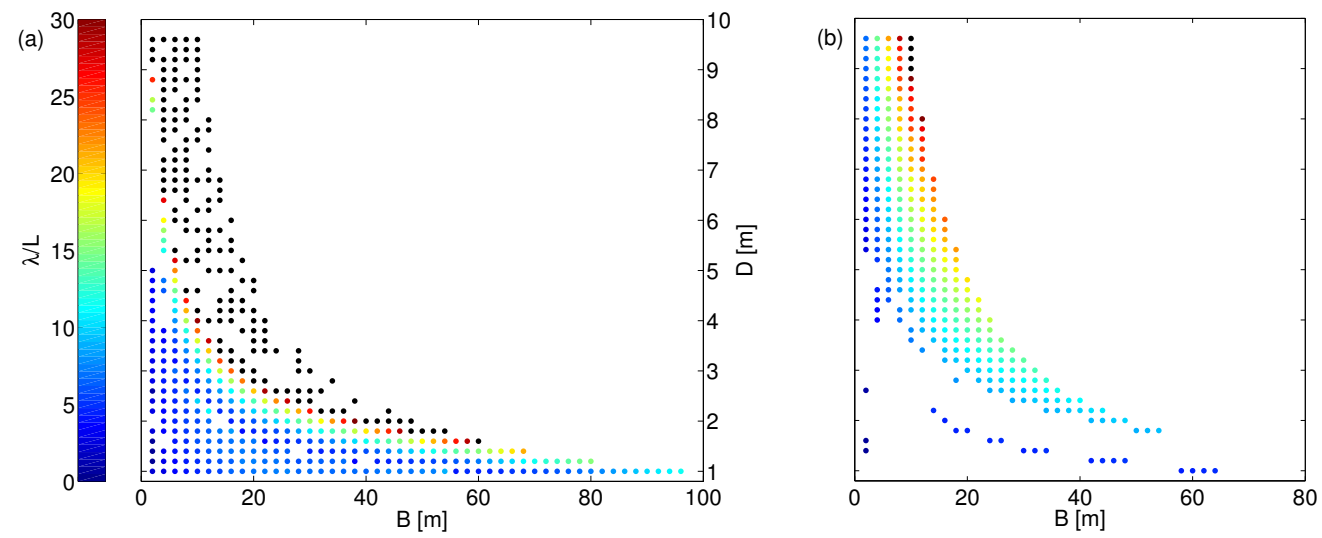

Figure 8: Wave length ratio corresponding to data that Fig. 4 was produced from.
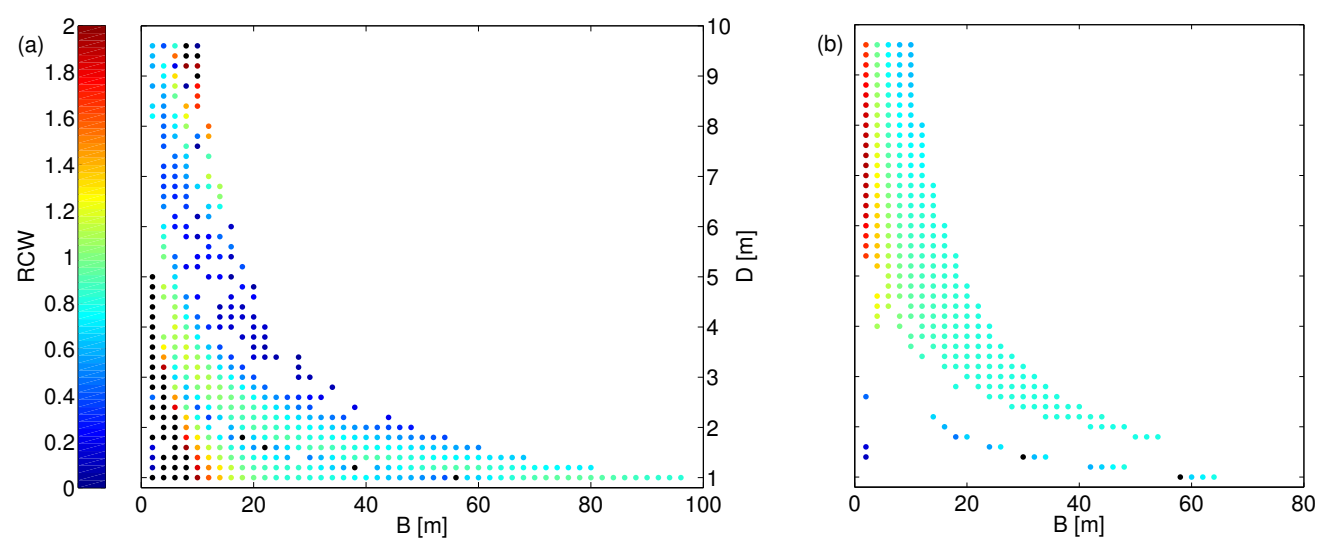

Figure 9: Relative capture width corresponding to data that Fig. 4 was produced from.

resolution of other features of interest.

\section{Discussion}

\subsection{Optimum approximately in resonance}

Relating Fig. 5 to Fig. 4 it can be said that maximal PTO (for any fixed $B$ and $D$ ) ${ }_{439}$ is due to resonance, at least in one of the two DOF's, because at least on of the frequency ${ }_{440}$ ratios approximates unity closely. (However, it is important that the frequency ratios are ${ }_{441}$ not exactly unity, as we explain this in Sec. 5.4.) The resonance peak is preserved even if the ${ }_{442}$ total power $P=P_{h}+P_{p}$ from heave and pitch is considered. The response in heave shows ${ }^{443}$ no interesting features: $P$ changes monotonically with $B$ or $D$; thus, features of the total ${ }^{444}$ PTO are inherited from pitch. (We demonstrate some behavior of the system that gives rise ${ }_{445}$ to this feature in Sec. 5.5.) However, it does not mean that heave is less productive. It is ${ }_{446}$ 
possible that $P_{p(h)}>P_{h(p)}$ while $\pi_{1(2)} \gg 1$ or $\pi_{1(2)} \ll 1$ and $\pi_{2(1)} \approx 1$. For both of the latter two points an illustration is provided by the individual of $(B, D)=(20,4.8)$, in which case resonance occurs in heave, and pitch slightly outperforms heave [cf. Fig. 4 (d) and (f)].

Comparing e.g. Figs. 6 (a) ((b)) and 4 (e) ((d)), features of the color charts for power are clearly related to that of the natural frequency. In heave, with decreasing $D$ the water plane area and so the stiffness increase, which increases the natural frequency. In pitch, the geometry determines the natural frequency in a nontrivial manner. Since the optimum is due to resonance, the performance the better the wave frequency the lower, as indicated by eq. (24).

\subsection{Hydrostatic stability}

Regarding the lower bounds on $z_{g}$ [Fig. 7 (b)], it is a simple inclined flat surface according to eq. (14). As for the upper bound [Fig. 7 (a)], it is rapidly growing for large $L$ 's according to eq. (13). Its excessively large values we chose not to resolve in the chart, because very high elevations of the COG are not practical, as it requires the density of the float to be very small. Nevertheless, the power is evaluated in accordance with the excessively large values of $z_{g}$. Clearly, imposing a smaller upper limit on $z_{g}$ would reduce the power. The optimal values of $z_{g}$ are searched for using fmincon within the limits implied by eqs. (14) and (13). When resonance occurs in heave, the lower limit is found always optimal [Fig. 7 (d)], and there is a departure from this at a certain point, already in the regime when resonance occurs in pitch [Fig. 7 (c)].

Figures 7 (a) and (b) prompt that very often the optimal $z_{g}$ is bracketed very tightly by its limiting values. Under such circumstances if the device oscillates, it is expected that - even if the requirements of hydrostatic stability are met - it may capsize. The critical wave height for capsizing can also be rather small. To prevent the WEC from capsizing a ballast or a keel to the lowermost point of the float can be installed. As an extension of the present analysis, the keel could be modeled as a concentrated point mass, with which the distribution of mass is nonuniform. The overall structural density would increase, and the device ballasted down would immerse deeper. The ratio of the mass of ballast and float could be a further nondimensional variable that the performance depends on, which would make the problem of optimization more complicated. In fact, the purpose of introducing variability in the density of the float was nothing but to maintain stability in case of any $(B, D)$ scenario; now, however, this solution turns out to be not always sufficient.

\subsection{Elevation of the COG impacting on the response bandwidth}

In our preliminary analysis using regular waves, in connection with Fig. $3, z_{g}$ was not optimized for, but held fixed at $z_{g}=0$. Some individuals featured in our analysis, subjected to appropriately narrow-banded irregular waves, turned out to have a nontrivial optimal $z_{g}$, however. In Fig. 10 featuring the individual of $D=2$ exposed to regular waves the effects of the increasing elevation of the COG is shown, resulting in: increasing response amplitude and decreasing bandwidth. For any particular $z_{g}>0$, through the inverted pendulum effect the natural frequency is smaller, at which frequency, for a fixed geometry, the frequencydependent damping is also smaller, and in the same time the excitation torque is greater. 


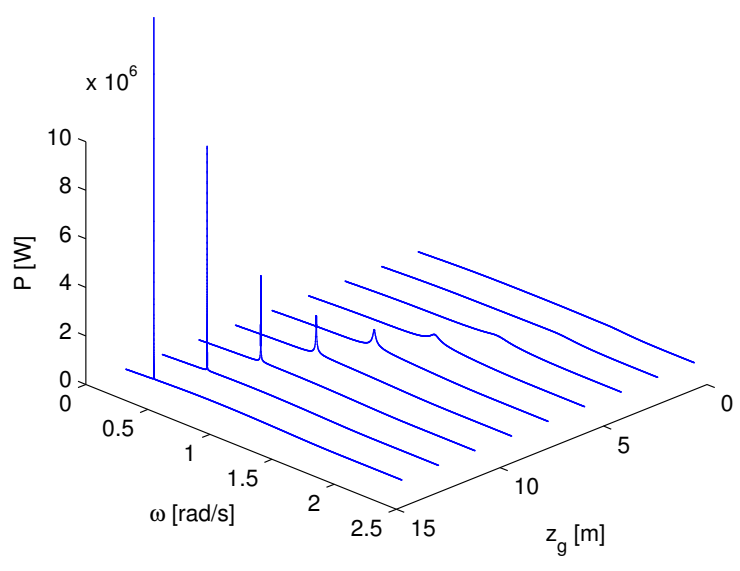

Figure 10: Total power take-off depending on the wave frequency and the elevation of the COG, for the individual $(B, D, L)=(5,2,20)$. The regular wave height was set to be: $H=2$.

\subsection{Optimum slightly off-resonant}

A similar situation to the latter is shown in Fig. 11 (a) for a wide ( $B=30)$ terminatortype WEC. Using irregular waves [see panel (b) of that figure] the lower limit for $z_{g}$ is found to be optimal, because the bandwidth of response is decreasing for the increasing elevation of the COG. For this optimal value, $z_{g, m i n}$, the response as a function of the frequency is reproduced in Fig. 12 (a). In the same diagram, for a comparison of the bandwidths, the spectrum of irregular waves is also shown (in green) with an arbitrary peak frequency. (The spectral ordinates $S(\omega)$ are scaled up appropriately in order to be able to present the spectrum in the diagram of $P\left(\omega_{0}\right)$.) As a gross feature the response is double-peaked; one peak corresponds with resonance in pitch (left), and another one with resonance in heave (right), the latter being a much more broadbanded effect. On a more detailed level, it can be seen that the peak corresponding to the resonance in pitch is double-peaked itself. The reason for this is the following. Figure 12 (b) shows the absolute value of the intrinsic impedance in pitch, $\left|Z_{i, p}\right|$, which is the optimal choice for the external damping $k_{p}$. It has a minimum value for the resonant frequency (which minimum value is not zero, just orders of magnitude smaller than the optimal $k_{p}$ well away from the resonant frequency). The bandwidth of the response $P_{1}$ depends on the impedance or damping (see Appendix B). Thus, the response $P_{1}$ is the most narrow-banded in resonance, and even though its ordinates 505 just off resonance are smaller, the bandwidth is larger, and so the synthesized response $P{ }_{506}$ with the use of irregular waves, according to eq. (16), can feature larger ordinates just off ${ }_{507}$ resonance.

\subsection{Nonmonotonic dependence of the power take-off in pitch}

For the geometry in question the excitation torque is also shown in Fig. 13, which is to 510 indicate that its magnitude depends on the frequency, and it has a characteristic maximum 511 at some point. This scenario is chosen from a ridge of the total power showcased in Fig. $4{ }_{512}$ 


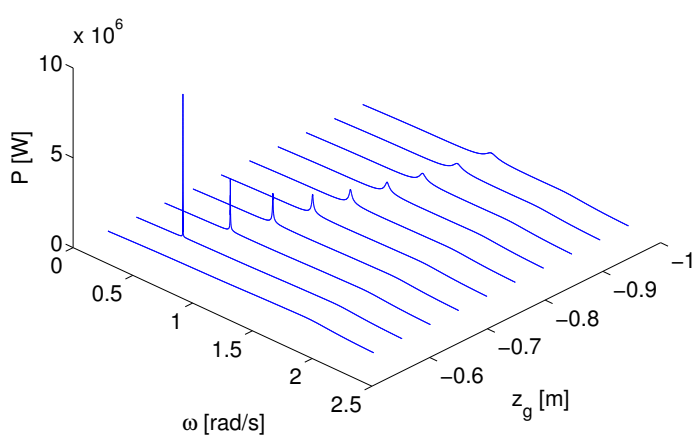

(b)

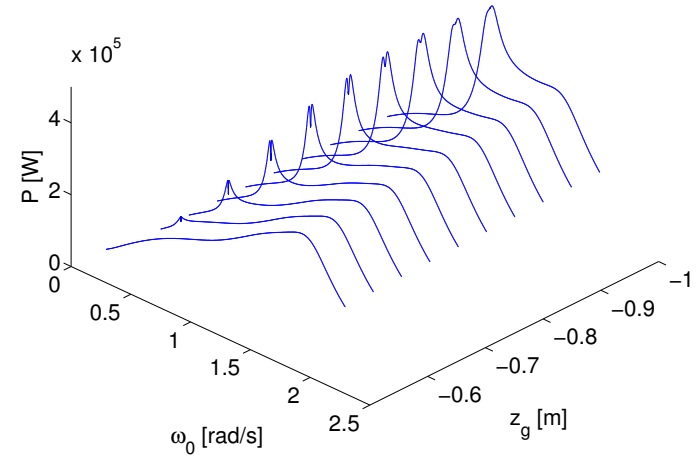

Figure 11: Total power take-off depending on the wave frequency and the elevation of the COG, for the individual $(B, D, L)=(3,2,3.2539)$, with regular waves of $H=2$ (a) and irregular waves of $H_{s}=2 \sqrt{2}$ (b).

(a) or (e) (corresponding to the maximum of power as a function of $D$ for each fixed $B$ ). It is pointed out that for $D=2$ the maximum of the excitation torque does not coincide with the resonance peak of the response $P$. A maximum of $P$ at $D=2$ occurs such that for smaller values of $D$ it is the magnitude of excitation that is decreasing, and for larger values of $D$, it is the resonant or natural frequency that is decreasing. For lower frequencies the magnitudes of excitation is smaller, even though its peak value is further increasing with $D$.

\subsection{Different characteristics of optimal power and $R C W$}

Similarly as with the power, the RCW also features a ridge [Fig. 9 (a)], but it does not coincide with that of the power. Instead, it seems to align with a curve in the $B$ - $D$ plane defined by $\pi_{1}=\pi_{2}=1$ (see Fig. 5), i.e., when resonance occurs in the two DOF's in the same time. (A strip closely confined to this curve shows up in isolation in the right hand side of all color charts.) This offset of the ridges is notable; it arises now because the frequency is a variable to be optimized for. On the ridge of the $\mathrm{RCW}$ the resonant frequencies in heave and pitch are equal, which value is greater than the optimal one. The lower-frequency wave 

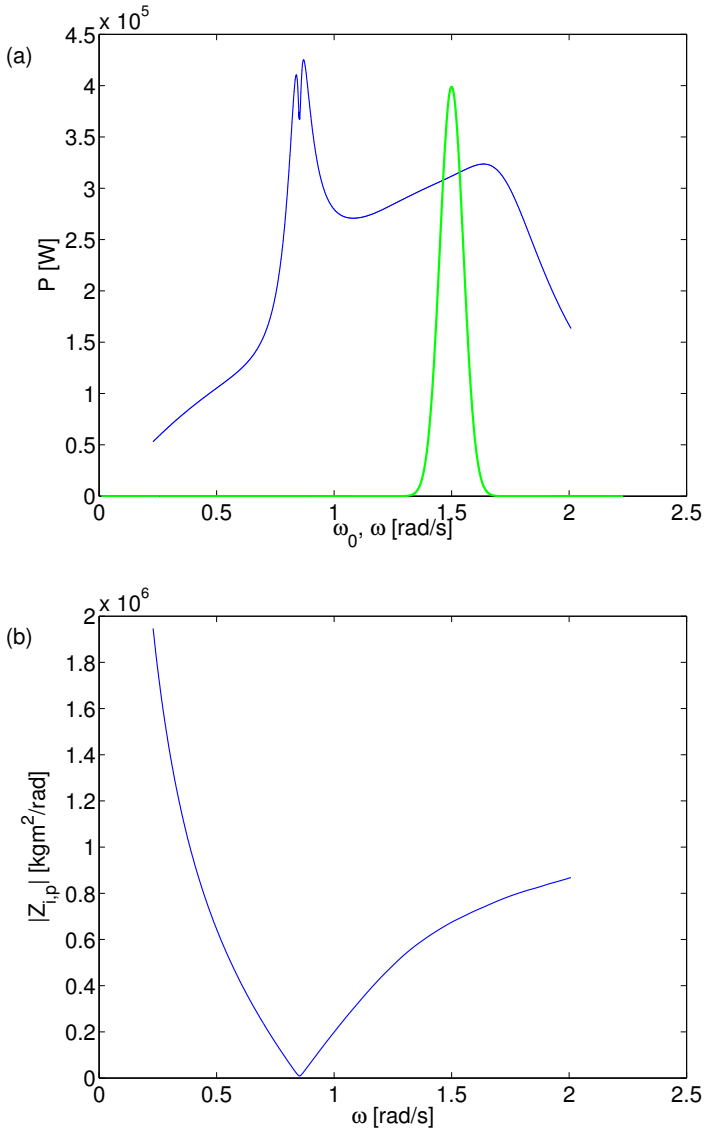

Figure 12: Showcase of an individual from the ridge of the surface of the total power take-off (PTO) seen in Fig. 4: $(B, D, L)=(3,2,3.2539)$. (a) Frequency response in terms of the total PTO. For reference, we included in the same diagram the model spectrum of irregular waves (thick green line) of an arbitrary peak frequency (ordinates are suitably scaled to fit the diagram). The irregular wave height is set as: $H_{s}=2 \sqrt{2}$; and $z_{g}=-0.8 \mathrm{~m}$. (b) Absolute value of the intrinsic impedance, which is not zero but has a minimum at resonance. 


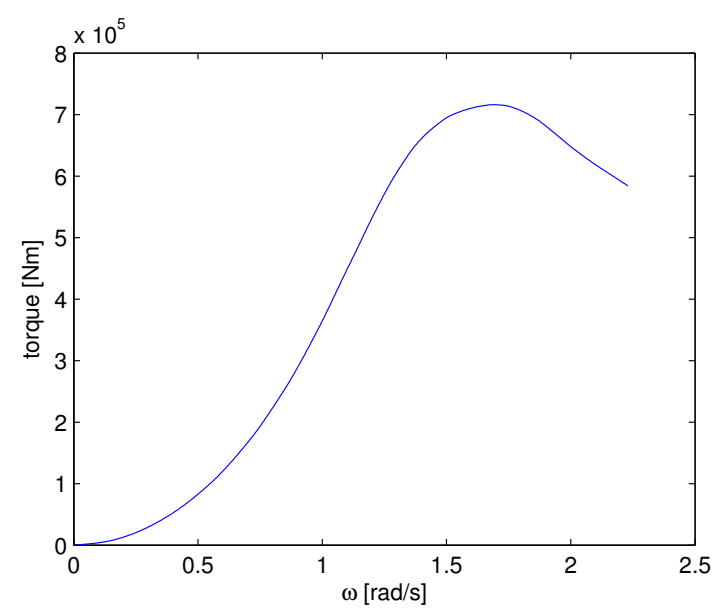

Figure 13: A follow up of Fig. 12: frequency dependent excitation torque in pitch.

carries more energy, and even if a smaller percentage is harnessed, the total performance can still be better. That is, interestingly, resonance in only one of the DOF's can be better than resonance in both DOF's.

Consider resonance in pitch. The color in Fig. 9 (a) indicates that the RCW approaches unity from below for increasing $B$ 's while fixing $L=L_{\text {min }}$. This is consistent with the fact that $B$ is an increasing multiple of $\lambda$ (see Fig. 8), that is, the 3D wave diffraction effects are more and more marginalized to the ends of the wide device, and so the situation is dominantly 2D. And since the situation is close to resonance in both DOF's, and the damping coefficients are set according to the amplitude criterion, we do indeed expect [14] that the incident waves are largely destructed. However, a full destruction of waves, even under the most favorable conditions, cannot happen, because of the finite bandwidth of the wave spectrum.

\subsection{Optimal geometry}

Reflecting on the performance characteristics of the concept (Fig. 4) we can say that optimal or well-performing individuals are found for extremal settings of the geometric parameters, which make either a very long barge head on the waves, an attenuator-type WEC, or a very long spine spanning as wide a window of the waves as possible, a terminatortype WEC. By changing the geometry in terms of $B$ and $D$, moving away from these extremes is the 'least costly' along the paths of either minimal $L$ or minimal $B$. Intermediate values of geometric parameters correspond to not so well-performing individuals.

\subsection{Optimal wavelength}

A realistic level of optimal performance can be associated with a very wide range of optimal wavelength ratios depending on the chosen geometry (Fig. 8); that is, in answer to an outstanding question concerning previous analysis: a universally applicable optimal 
value cannot be identified. For better-performing longer attenuator-type WEC's, the optimal ${ }_{551}$ wavelength is comparable with the device length, being typically a few multiples of it. $\quad{ }_{552}$

It is emphasized once more that in our analysis the frequency is optimized for. It is 553 consistent with an approach when first a well-performing WEC is designed, and then suitable ${ }_{554}$ wave sites are searched for. In the reverse direction a particular wave site has to be utilized 555 and best accommodated, the environmental conditions are thus being given. This way the 556 frequency is not a variable but fixed; or more realistically, instead of a single frequency value, ${ }_{557}$ a so-called wave scatter (wave height-wave period) matrix describing the conditions at the ${ }_{558}$ particular site is given. This may change the solution to the problem as provided by Fig. $4 .{ }^{559}$

\section{Summary and concluding remarks}

In this paper we advanced previous linear time-domain analysis of a large box barge, which is exposed to significant wave diffraction forces when its dimension along the direction of wave propagation is comparable to the wavelength, i.e., when the wavelength ratio - a measure introduced to the analysis of wave energy converters (WEC) by Kraemer - is small. Kraemer reported, however, that, considering regular waves, in order to achieve larger response of the free-floating box barge in pitch (regarded favorable in case of WECs) the wavelength ratio does not need to be small or near unity, but can be much larger than that. Here we conducted a similar analysis using a nonlinear time-domain model, and found an optimal value of about five. Such an optimum emerges due to the progressively, i.e., nonlinearly, increasing buoyancy restoring force with increasing angular displacement and so oscillation amplitudes.

We found also another mechanism that can create an optimum: with increasing wavelength ratio the bandwidth of the transfer function is decreasing progressively, and so the WEC is increasingly incapable of responding to irregular waves. That is, with irregular waves (of moderate height) the oscillation amplitudes remain in the approximately linear regime. Therefore, also considering that calculations in the frequency-domain are much more straightforward and inexpensive, we preferred for our analysis to use linear frequency-domain models with irregular waves.

As the subject of our new and main analysis, we proposed a simple wave energy converter concept by adding dampers to the box barge in the degrees of freedom of pitch and heave, linking it to the mechanical reference. We showed that in a linear frequency-domain model these degrees of freedom decouple, and so the power take-off is made up of two independent parts. In this favorable case we were able to develop a customized method of optimum search, and to apply it successfully. For the algorithmic optimization of the wave frequency and the elevation of the center of gravity we exploited the simplicity of the model, which allowed us to derive the response analytically; and for the determination of the optimal values of the damping coefficients we applied theory, namely, the amplitude criterion of optimum. We conducted the optimum search in terms of dimensional variables, and then translated the results into terms of nondimensional variables for the purpose of physical interpretation: For any geometry, we found optimum approximately in resonance, occurring at least in one of the two degrees of freedom. 
We have identified two geometrical parameters, the breadth and draught of the box barge, with respect to which there is no unique optimum in the sense of a local maximum of the power take-off occurring at some nontrivial intermediate value of any of the parameters. As an algorithmic optimization procedure concerning the breadth and draught could not provide immediately meaningful results, the dependence of the performance and other quantities on these two parameters is provided here visually by color charts. The chart of the performance revealed that better performing individuals have extremal geometry: it is either a long attenuator-type device, or a wide terminator-type device, with dominant contribution from pitch or heave, respectively. As for the former type, the optimal wavelength (deriving from the optimal frequency) is comparable with the optimal device length (conditioned to a fixed device size/displacement, and so restricted through the restrictions on $D$ and $B$ imposing some minimal values on them) - typically a few multiples of it - and is shorter if the bandwidth of the irregular wave frequency is broader. In this regard we note that diffraction effects are more significant considering optimal performance in irregular seas over the previously studied optimal - or, putting it more precisely: most intensive - motion (damped only through wave radiation) due to regular waves.

We have also found that - similarly as with the wave length ratio described above - the response amplitude (bandwidth) of the transfer function increases (decreases) monotonically with the elevation of the center of gravity of the barge in the linear model with regular waves. Again, this is rendered by either nonlinear progressive restoring forces or irregular waves. Through these two mechanisms an optimal elevation can emerge.

Some remarks on the validity of the results produced by our simple model are due here, however. Excessively large predicted optimal oscillation amplitudes and/or large wave heights are not consistent with the linear diffraction analysis - apart from the fact that nonlinear buoyancy forces are already accounted for. In this case performing simulations by solving the fundamental equations of fluid mechanics or empirical model tests (in laboratory or at sea) might be necessary.

Even if the oscillations are within the linear regime to a good approximation, they might be still large enough that the device would capsize, which is an event that our frequencydomain model cannot register. Having performed the optimization with the linear model, therefore, a reality check must follow, e.g. using the time-domain model built in AQWANAUT to check for the possibility of capsizing in the case of suspected individuals. It is expected in general that theoretically optimal and robust operational conditions rarely coincide, and a compromise has to be made. Nevertheless, linear models can be extremely powerful as a guide for the optimum search, or, the search for viable concepts and individuals.

The much more difficult problem of finding an economic optimum has not been pursued here. For this the costs have to be modeled in terms of the device size.

\section{Acknowledgements}

Facilities to carry out this research were provided by Green Ocean Energy Limited (UK); it is gratefully acknowledged. 


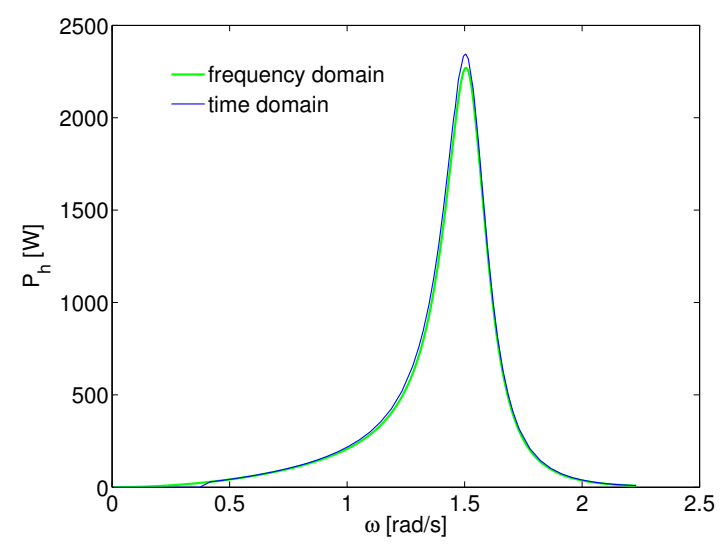

Figure A.14: Comparison of simulation results, in terms of the power generated in heave, for a frequencydomain and a time-domain model, using very small waves $(H=0.1)$. The scenario is described by the following: $(B, D, L)=(30,3.2,2.0337)$.

\section{Appendix A. Agreement of the linear frequency-domain and nonlinear time- ${ }_{632}$ domain models

The validity of the linear model of our box-hulled WEC concept breaks down when ${ }^{634}$ excessively large amplitudes of excursion are predicted, typically in resonance. For small ${ }_{635}$ amplitude oscillations the linear model is accurate. Its predictions in terms of the power ${ }_{636}$ in heave are corroborated with the predictions of the nonlinear model, as shown in Fig. ${ }^{637}$ A.14. The regular wave height is set to be $H=0.1$. The damping is held constant, at a ${ }_{638}$ value which is optimal for the resonant natural frequency. The time-domain model involves ${ }_{639}$ an arm of length $l_{a}=50 \mathrm{~m}$, which, using very small waves, is approximately the same in ${ }_{640}$ effect as a vertical slide. For somewhat larger amplitudes the finite arm length has a strong ${ }_{641}$ effect on the performance both in heave and pitch (not shown here); and it also makes a ${ }_{642}$ significant difference if it is a leading or trailing arm (equivalent in effect with reversing the ${ }_{643}$ wave direction, also not shown).

\section{Appendix B. Growing response amplitude with vanishing bandwidth ${ }_{645}$}

For a 1-DOF harmonic oscillator it is easy to show that the resonant velocity amplitude ${ }_{646}$ $\left(u_{\max }\right)$ can be expressed in terms of the amplitude of excitation force $(\mathrm{F})$ and the damping ${ }_{647}$ $(R$, including internal and external damping) as follows:

$$
u_{\text {max }}=F / R .
$$

This relation is verified in case of the individuals considered in connection with Fig. $2{ }_{649}$ by evaluating terms on the left and right hand sides. Motion in the independent DOF of ${ }_{650}$ pitch is considered. On the one hand we obtained $u$ by using the formula derived for the ${ }_{651}$ displacement response [analogous to (2)]. The displacement is multiplied by the frequency ${ }_{652}$ 
$\omega$ to have velocity. Using discrete values of the frequency, with a sufficiently fine resolution, the maximal velocity is selected. On the other hand we took the fraction of the force and damping as obtained by radiation-diffraction analysis. Diagrams of the frequency-dependent force coefficient and radiation damping are shown in Fig. B.15 (a) and (b), respectively. The curves are coded by the same color as the markers on the horizontal axis that indicate by their position on the axis the corresponding natural frequency. It can be seen that with increasing draughts (device size fixed) the excitation torque is decreasing, and, on the other hand, the radiation damping is decreasing at an even greater rate. This is true in terms of either the peak values that these frequency-dependent functions take, or the values taken at the natural frequencies. When the data obtained by the two alternative means are plotted in the same diagram, Fig. B.16 (b) concerning the free-floating box barge, the agreement appears to be exact. Thus, a monotonically growing response amplitude with variation in the geometry is thereby accounted for.

In the same linear framework it is meaningful to define the bandwidth and relative bandwidth [14], respectively, of a 1-DOF linear oscillatory system as follows:

$$
\Delta \omega=R / m
$$

and

$$
\Delta \omega / \alpha=R / \sqrt{s m} .
$$

The meaning of $\Delta \omega$ is a frequency range where the kinetic energy exceeds half of its maximal value $\left(m u_{\max }^{2} / 4\right)$; and $\alpha$ 'refers to' the resonant or natural frequency. The relative bandwidth (which is the inverse of the nondimensional velocity amplitude) has been evaluated for the considered individuals, and the resulting diagram is plotted in Fig. B.16 (a). Indeed, in parallel with increasing (dimensional) amplitude, the bandwidth is vanishing. This, accounts for our observations regarding Fig. 2 (a).

\section{References}

[1] Callaghan J. Future Marine Energy. Tech. Rep. CTC601; Carbon Trus; 2006. Available from: www.carbontrust.co.uk.

[2] Falnes J. A review of wave-energy extraction. Marine Structures 2007;20(4):185 - 201.

[3] Kraemer DRB. The effect of wavelength on the response of floating ocean wave-energy conversion devices. In: Proceedings of the 7th European Wave and Tidal Energy Conference. Porto, Portugal; 2007 .

[4] Iversen LC. Numerical method for computing the power absorbed by a phase-controlled point absorber. Applied Ocean Research 1982;4(3):173 -80.

[5] Eidsmoen H. Optimum control of a floating wave-energy converter with restricted amplitude. J Offshore Mech Arct Eng 1996;118:96-102.

[6] Korde UA. Latching control of deep water wave energy devices using an active reference. Ocean Engineering 2002;29(11):1343 -55.

[7] Babarit A, Duclos G, Clment A. Comparison of latching control strategies for a heaving wave energy device in random sea. Applied Ocean Research 2004;26(5):227 -38.

[8] Babarit A, Clément AH. Optimal latching control of a wave energy device in regular and irregular waves. Applied Ocean Research 2006;28(2):77-91. 

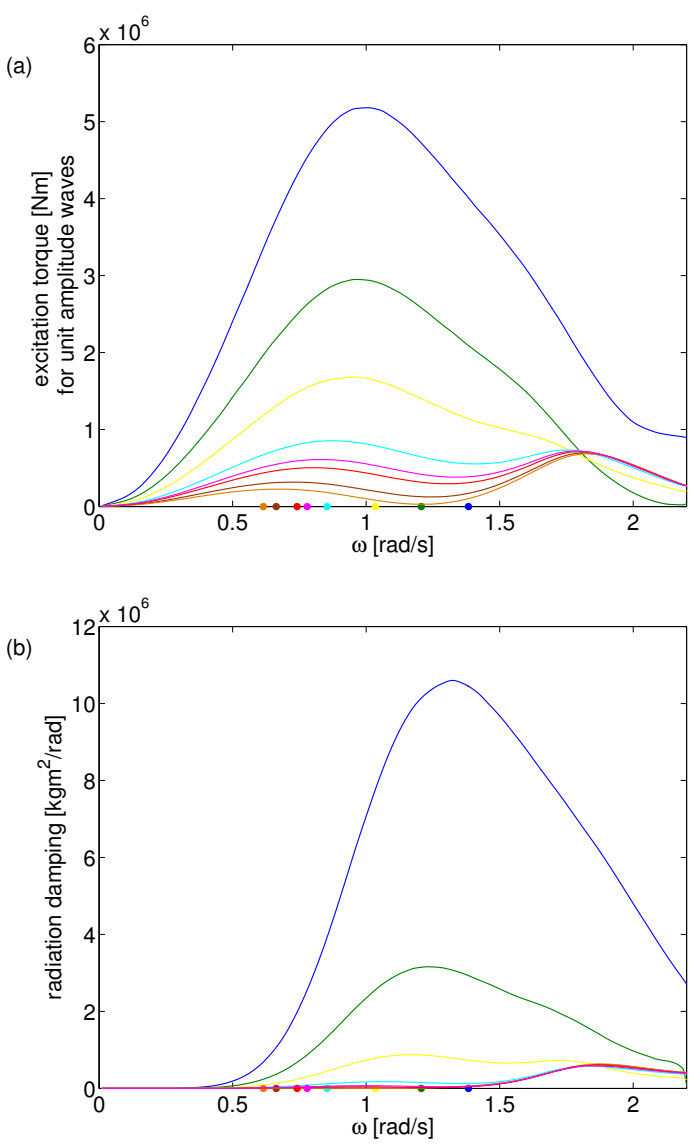

Figure B.15: Hydrodynamic parameters related to features of the diagrams seen in Fig. 2. In each diagram the same individuals are considered as in the said figure. The colored markers on the horizontal axis of frequency mark the values of the natural frequencies belonging to the curves of the respective hydrodynamic property each drawn in the same color as the marker. The markers from right to left in order correspond to the curves from top to bottom. 


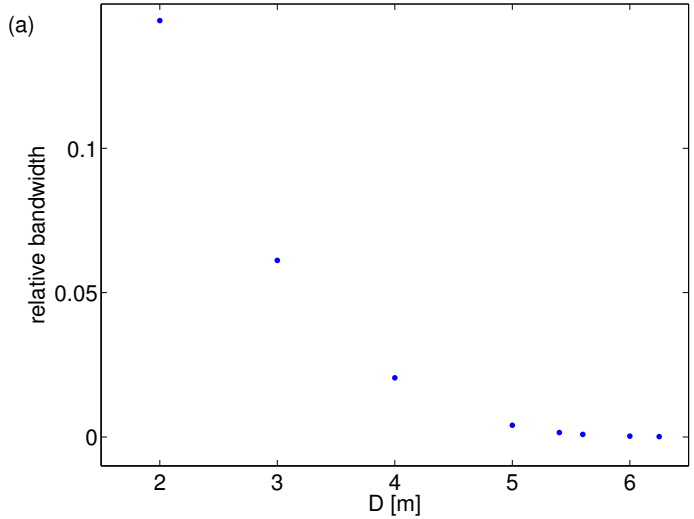

(b)

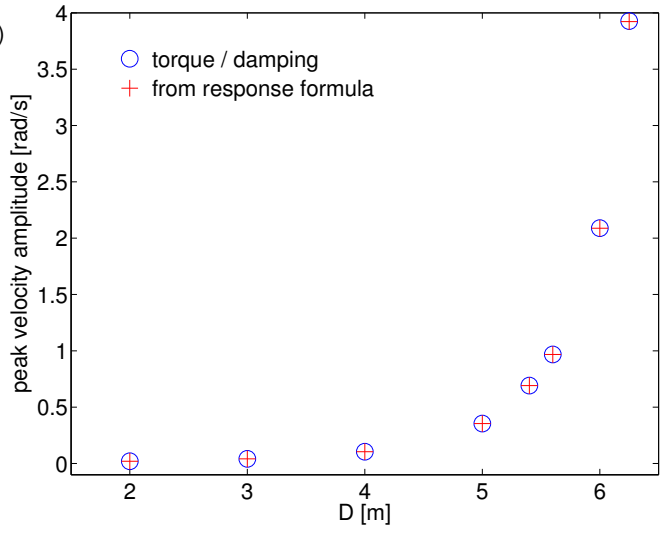

Figure B.16: A follow up of Fig. B.15. In each diagram the same individuals are considered as in the said figure. In panel (b) the cross marker shows values obtained by a response formula analogous to (2). 
[9] Kara F. Time domain prediction of power absorption from ocean waves with latching control. Renewable 692 Energy 2010;35(2):423 -34.

[10] Hong Y, Waters R, Boström C, Eriksson M, Engström J, Leijon M. Review on electrical control strategies for wave energy converting systems. Renewable and Sustainable Energy Reviews 2014;31(0):329-42.

[11] Cerveira F, Fonseca N, Pascoal R. Mooring system influence on the efficiency of wave energy converters. International Journal of Marine Energy 2013;3-4(0):65-81.

[12] Zanuttigh B, Angelelli E, Kofoed JP. Effects of mooring systems on the performance of a wave activated body energy converter. Renewable Energy 2013;57(0):422 -31.

13] Aurelien D, Babarit A, Clément AH. Optimizing the power take off of a wave energy converter with regard to the wave climate. J Offshore Mech Arct Eng 2005;128:56-64.

[14] Falnes J. Ocean waves and oscillating systems. Cambridge University Press; 2002.

[15] Evans DV. Power from water waves. Annual Review of Fluid Mechanics 1981;13(1):157-87.

[16] Salter SH. Wave power. Nature 1974;249:720-4.

17] Evans DV, Jeffrey DC, Salter SH, Taylor JRM. Submerged cylinder wave energy device: theory and experiment. Applied Ocean Research 1979;1(1).

[18] Korde U. On providing a reaction for efficient wave energy absorption by floating devices. Applied Ocean Research 1999;21(5):235 -48.

19] Sonin AA. The physical basis of dimensional analysis (Second Edition). Department of Mechanical 709 Engineering, MIT, Cambridge, MA 02139; 2001.

[20] Evans D. A theory for wave-power absorption by oscillating bodies. Journal of Fluid Mechanics $1976 ; 77: 1-25$.

[21] Count BM. On the dynamics of wave-power devices. Proceedings of the Royal Society of London Series A, Mathematical and Physical Sciences 1978;363(1715):559-79.

[22] Garrison CJ. Hydrodynamic Loading of Large Offshore Structures: Three-dimensional Distribution Methods. Wiley; 1978,

[23] Kemp JF, Barrass CB, Young P. Ship Stability: Notes and Examples. Butterworth-Heinemann; third ed.; 2001.

[24] Chakrabarti SK. Hydrodynamics of Offshore Structures. WIT Press; 1987.

[25] Barltrop NDP. Dynamics of Fixed Marine Structures. Marine Technology Directorate Ltd.; 1991.

[26] Tucker MJ, Pitt EG. Waves in Ocean Engineering; vol. 5. Elsevier, Kidlington, UK; 2001. 